\title{
Bericht über die XIII. internationale Wissenschaftskonferenz: „Der Weltjugendtag. Ursprung und Entwicklung der Idee" Krakau, 1. und 2. April 2016
}

Der Weltjugendtag (Światowe Dni Młodzieży: ŚDM) ist ein bedeutendes Ereignis mit vielerlei Aspekten, bei dem sich geistige und religiöse Erlebnisse mit den Bereichen des gegenseitigen Kennenlernens, von Kultur, Gesellschaft, Pädagogik, Erziehung und Tourismus verknüpfen. Im Rahmen der direkten Vorbereitung auf den Weltjugendtag in Krakau fand am 1. und 2. April 2016 in eben dieser Stadt die internationale Konferenz „Światowe Dni Młodzieży. Źródła i rozwój idei" (Der Weltjugendtag. Ursprung und Ideenbörse) statt. Die Schirmherrschaft übernahmen Seine Eminenz Stanisław Kardinal Dziwisz, Metropolit in Krakau, Seine Magnifizenz H. H. Prof. Dr. habil. Wojciech Zyzak, Rektor der Päpstlichen Universität Johannes Paul II. in Krakau (UPJPII) sowie Seine Magnifizenz Prof. Dr. habil. Wojciech Nowak, Rektor der Jagiellonen-Universität (UJ). Die Konferenz wurde von zwei Hochschulen aus Krakau organisiert: von der Päpstlichen Universität Johannes Paul II. in Krakau und der Jagiellonen-Universität in Krakau. Die Initiative dazu entstand in den akademischen Kreisen beider Universitäten, deren Vertreter beim Außenministerium der Republik Polen das Projekt „Współpraca w dziedzinie dyplomacji publicznej 2016” (Zusammenarbeit in der öffentlichen Diplomatie 2016) eingereicht hatten, das dann auch genehmigt wurde. Folgende Stiftungen unterstützten und finanzierten die Konferenz mit: Instituto Acton, Fondazione de Gasperi sowie Fondazione Internazionale Giovanni Paolo II per il Magistero Sociale della Chiesa. Inspirationen für die Unternehmung boten die Symbolik und die Bedeutung 
des Ortes, den Papst Franziskus für den Weltjugendtag gewählt hatte. Mit seiner Entscheidung führte er die Idee gewissermaßen zurück nach Polen, wo im Jahr 1991 der erste Weltjugendtag in Tschenstochau stattgefunden hatte. Insbesondere steht natürlich die Stadt Krakau in engem Bezug zu Johannes Paul II., dem Begründer dieses globalen Ereignisses, das nicht nur religiöse sondern auch internationale und kulturelle Aspekte vereint.

Die Konferenz versammelte viele bedeutende Gäste und Referenten, darunter unter anderem: H. H. Kardinal Stanisław Dziwisz, H. H. Kardinal Stanisław Ryłko, Erzbischof Celestino Migliore, Marcello Bedeschi, Danny Cassey, Kishore Jayabalan, Mimmo Muolo, Prof. Yago de la Cierva Alvarez de Sotomayor, Schwester Nathalie Becquart, sowie weitere Vertreter der Wissenschaft aus Polen, die den Weltjugendtag unter dem Gesichtspunkt ihrer jeweiligen Disziplin untersuchten. Darüber hinaus waren Wissenschaftler und Religionsführer, Teilnehmer und Organisatoren der bisherigen Weltjugendtage anwesend: aus Rom (1985, 2000), Buenos Aires (1987), Santiago de Compostela (1989), Tschenstochau (1991), Denver (1993), Manila (1995), Paris (1997), Toronto (2002), Köln (2005), Sydney (2008), Madrid (2011), daneben auch alle, die sich bei der Vorbereitung der Jugend auf den diesjährigen Weltjugendtag in Krakau (2016) engagierten. Das Hauptziel der Konferenz bestand darin, die Quellen für die Inspiration und die ausgesendeten Signale der Weltjugendtage im religiösen, gesellschaftlichen, kulturellen sowie internationalen Kontext zu erkennen. Auf diese Weise wurde sie zu einer weiteren Etappe auf dem Weg der intellektuellen und geistigen Vorbereitung auf die Begegnung der Jugend aus der ganzen Welt mit dem Heiligen Vater Franziskus. Die internationale Konferenz richtete sich unter anderem an folgenden Personenkreis: an zukünftige Teilnehmer sowie Mitglieder des Freiwilligendiensts am Weltjugendtag in Krakau, an Studenten und Dozenten der unterschiedlichen Fachbereiche, an Journalisten und Mitarbeiter der Medien, aber auch an Interessierte aus anderen Ländern, denen sich die Gelegenheit bieten wird, den gesellschaftlich-historischen Kontext des akademischen und wissenschaftlichen Krakaus kennen zu lernen.

Der erste Konferenztag (1. April) gliederte sich in die Inauguration des neuen Semesters sowie in drei Hauptsitzungen. Dabei wurde die Inauguration von H. H. Prof. Dr. habil. Józef Stala geleitet, dem Prorektor der UPJPII und Vorsitzenden des Organisationskomitees dieser Konferenz. In seiner Einführung begrüßte er die Rektoren der UPJPII, H. H. Prof. Dr. habil. Wojciech Zyzak, sowie der UJ, Prof. Dr. habil. med. Wojciech Nowak. Prof. J. Stala verlas den Versammelten das besondere Schreiben - mit dem Segen von Papst 
Franziskus, das der apostolische Nuntius in Polen, Erzbischof Celestino Migliore, Kardinal St. Dziwisz übergeben hatte. Anschließend präsentierte Jerzy Chmielewski, Botschafter und Bevollmächtigter des Außenministeriums in Sachen des Weltjugendtags, den Brief des Außenministers Polens, Dr. Witold Waszczykowskis, den dieser an die Organisatoren der Konferenz gerichtet hatte. Es folgte das Grußwort des Präsidenten der Stadt Krakau, Prof. Dr. habil. Jack Majchrowski, das Paweł Stańczyk, Sekretär der Stadt Krakau, vortrug. Als Überleitung in den Vortragsteil führten Erzbischof Celestino Migliore, Kardinal Stanisław Dziwisz und Kardinal Stanisław Ryłko in die Thematik um den Weltjugendtag ein. Dabei verwiesen sie auf dessen aktuelles Verständnis, die übermittelte Botschaft, aber auch auf seine Entstehungsgeschichte und die Entwicklung, wie diese aus Sicht des Päpstlichen Rats für Laien gesehen wird.

In der ersten Sitzung „ŚDM - rozwój idei” (Der Weltjugendtag Entwicklung der Idee), die Prof. Dr. habil. Bogdan Szlachta (Dekan der Fakultät für Internationale und Politische Studien an der UJ) leitete, ging es um einen historischen Abriss des Weltjugendtags, seine geistige Dimension, die Bedeutung der Kommunikation, den Bezug zum Weltjugendtag in Sydney sowie ein angemessenes Erleben der Freiheit. Die zweite Beratung „ŚDM - retrospekcja 1985-2013" (Der Weltjugendtag - eine Retrospektive 1985-2013) gestaltete sich als Podiumsdiskussion, die Dr. Krzysztof Strzałka (UJ) moderierte. Dabei wurden Vorbereitungen, Organisation, Verlauf und Events der Städte vorgestellt, die bereits als Gastgeber für den Weltjugendtag fungiert hatten. Die anschließende dritte Sitzung „ŚDM Kraków 2016” (Der Weltjugendtag 2016 in Krakau) beschäftigte sich unter der Leitung von H. H. Grzegorz Suchodolski (Organisationskomitee des Weltjugendtags, Krakau) mit dem diesjährigen Weltjugendtag in Krakau. Dabei stand der Gedanke des Heiligen Vaters Franziskus im Mittelpunkt, dass die Barmherzigkeit für die Glaubwürdigkeit des Christen in der modernen Welt stehe. H. H. Bischof Dr. Damian Muskus (Vorsitzender des Organisationskomitees des Weltjugendtags in Krakau) begann mit dem Referat „Miłosierdzie jest sprawdzianem wiarygodności chrześcijan we współczesnym świecie (Franciszek). ŚDM Kraków 2016 jako szkoła Bożego i ludzkiego miłosierdzia" (Die Barmherzigkeit macht die Glaubwürdigkeit des Christen in der modernen Welt aus [Franziskus]. Der Weltjugendtag 2016 in Krakau als Schule der göttlichen und der menschlichen Barmherzigkeit). Nach intensiven, ganztägigen Beratungen sowie der von H. H. Dariusz Raś hervorragend organisierten Bewirtung konnten die Teilnehmer der Konferenz in der Basilika St. Maria ihre wissenschaftlichen Diskurse mit Gebet und Liturgie 
verbinden. Als Hauptzelebrant der Messe und Prediger konnte H. H. Kardinal Stanisław Ryłko gewonnen werden.

Am zweiten Konferenztag (2. April) wurde während der vierten Sitzung „ŚDM jako przedmiot refleksji naukowej” (Der Weltjugendtag als Objekt wissenschaftlicher Reflexionen) unter der Leitung von H. H. Prof. J. Stala (UPJPII) der Weltjugendtag als Gegenstand mannigfaltiger wissenschaftlicher Reflexionen beleuchtet. Die polnischen Wissenschaftler betrachteten den Weltjugendtag unter folgenden Gesichtspunkten: Der Weltjugendtag im Hinblick auf die weltweiten Erfahrungen (Jagiellonen-Universität: Prof. Dr. habil. Antoni Jackowski, Dr. habil. Elżbieta Bilska-Wodecka, Dr. habil. Izabela Sołjan, Edyta Kostrzewa, Magister Justyna Liro, Magister Maciej Trojnar), als Bildungsmodell für die Jugend (Schlesische Universität: Dr. Elżbieta Tkocz), als aktuelle Antwort auf das Bedürfnis nach zeitgemäßen Feierlichkeiten (Katholische Universität in Lublin: Dr. Anna Sugier-Szerega), als religiöses Festival (Universität Stettin: H. H. Dr. habil. Andrzej Draguła, Prof. US) sowie als Element des Projekts einer christlichen Lebensführung (Nikolaus-Kopernikus-Universität: Dr. Michał Białkowski). Dabei wurden jedoch auch die Schwierigkeiten und Herausforderungen unterstrichen, den Glauben in der heutigen, wenig religiös geprägten Zeit zu bekennen (Dr. habil. Elżbieta Osewska, Prof. PWSZ ). Wie bereits am ersten Tag hatten die Konferenzteilnehmer auch am zweiten Tag reichlich Gelegenheit, sich an der lebhaften Diskussion zu beteiligen.

Nach Beendigung der Beratungen des zweiten Tags besichtigten die Besucher der Konferenz das älteste Gebäude der Jagiellonen-Universität - das Collegium Maius - und pilgerten auf den Spuren des Papstes aus Polen nach Wadowice, in das Museum im Elternhaus des hl. Johannes Paul II.

Der internationalen Konferenz war eine Pressekonferenz vorausgegangen. Während der beiden Konferenztage wurden etwa 300 Teilnehmer gezählt, Vorträge von 35 Referenten gehalten, darunter 12 aus Ländern außerhalb Polens. Die Referenten repräsentierten 16 akademische Zentren und weitere Institutionen. Alle Redebeiträge der Konferenz wurden simultan in 3 Sprachen übersetzt: ins Polnische, Englische und Italienische. Dem Organisationskomitee gehörten an: H. H. Prof. Dr. habil. Józef Stala - Vorsitzender des Organisationskomitees Prorektor der UPJPII; von der Jagiellonen-Universität: Dr. habil. Andrzej Porębski - Prodekan der Fakultät für Internationale und Politische Studien UJ; Dr. Małgorzata Stefanowicz; Dr. Krzysztof Strzałka; Dr. Paweł Plichta; von der Päpstlichen Universität Johannes Paul II.: Iwona Bąk - Leiterin des Zentrums für wissenschaftliche Untersuchungen und internationale Zusammenarbeit; 
Tomasz Kniaź; von der Metropolitenkurie in Krakau: H. H. Tomasz Szopa, Sekretär des Metropoliten Krakaus. Darüber hinaus engagierten sich viele Volontäre der UPJPII sowie der UJ.

Es ist zu betonen, dass die Konferenz zusätzlich zu einem tiefer gehenden Bewusstsein für die christliche Identität und Tradition Polens beigetragen hat sowie für dessen Beitrag zur europäischen, christlich-abendländischen Kultur und Zivilisation, insbesondere im Kontext des Jubiläums der Christianisierung Polens vor 1050 Jahren. Die Konferenz ermöglichte es den Teilnehmern, solides Wissen über das Land zu gewinnen, in das wenige Monate später Jugendliche aus der gesamten Welt reisen würden, besonders aus mit dem Projekt befassten Ländern. Während der Diskurse konnten Erfahrungen, Materialien und Texte gesammelt werden, mit deren Hilfe die Teilnehmer des Weltjugendtags in Krakau die gesellschaftlichen und religiösen Erfahrungen in Polen an der Schwelle vom XX. zum XXI. Jahrhundert besser verstehen werden. Denn diese stehen in enger Verbindung mit dem Wirken und der Lehre des hl. Johannes Paul II. und nehmen bis jetzt bedeutenden Einfluss auf die Zukunft Polens, des europäischen Kontinents und der gesamten Welt.

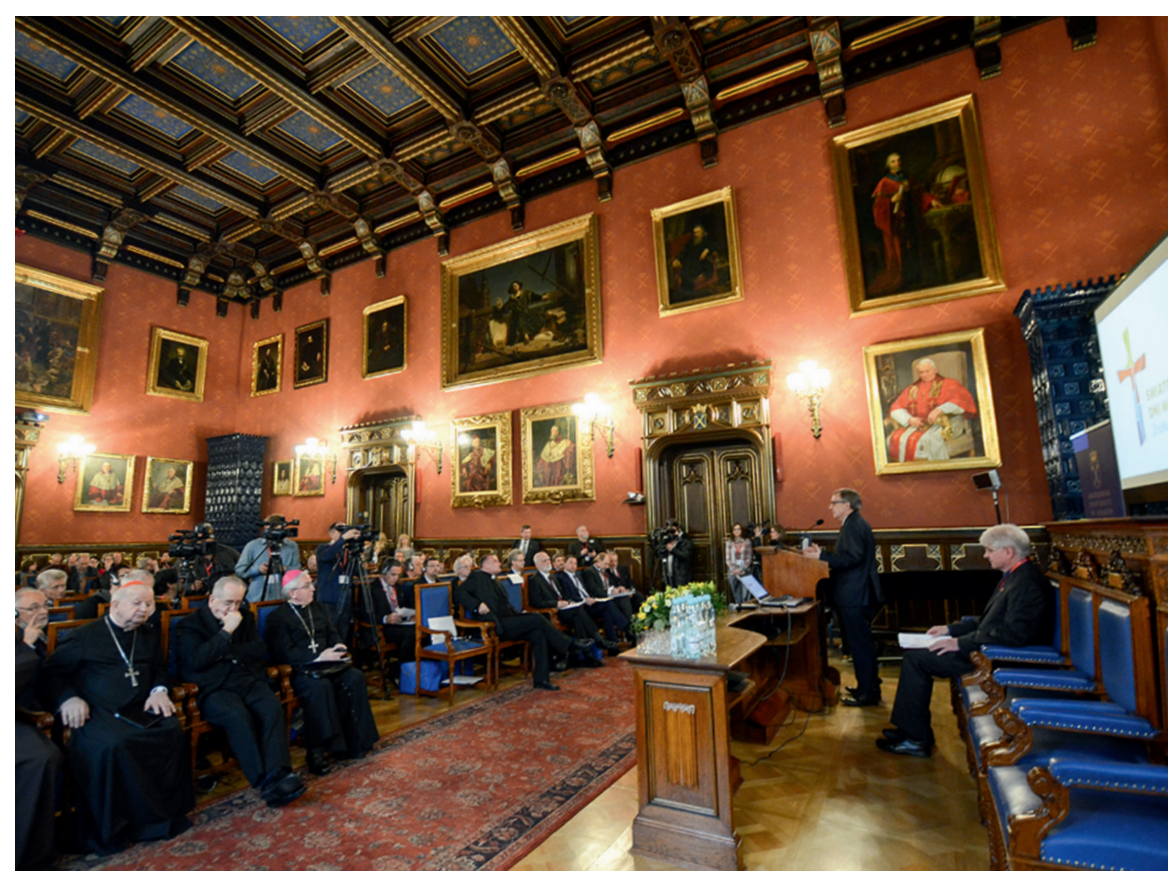



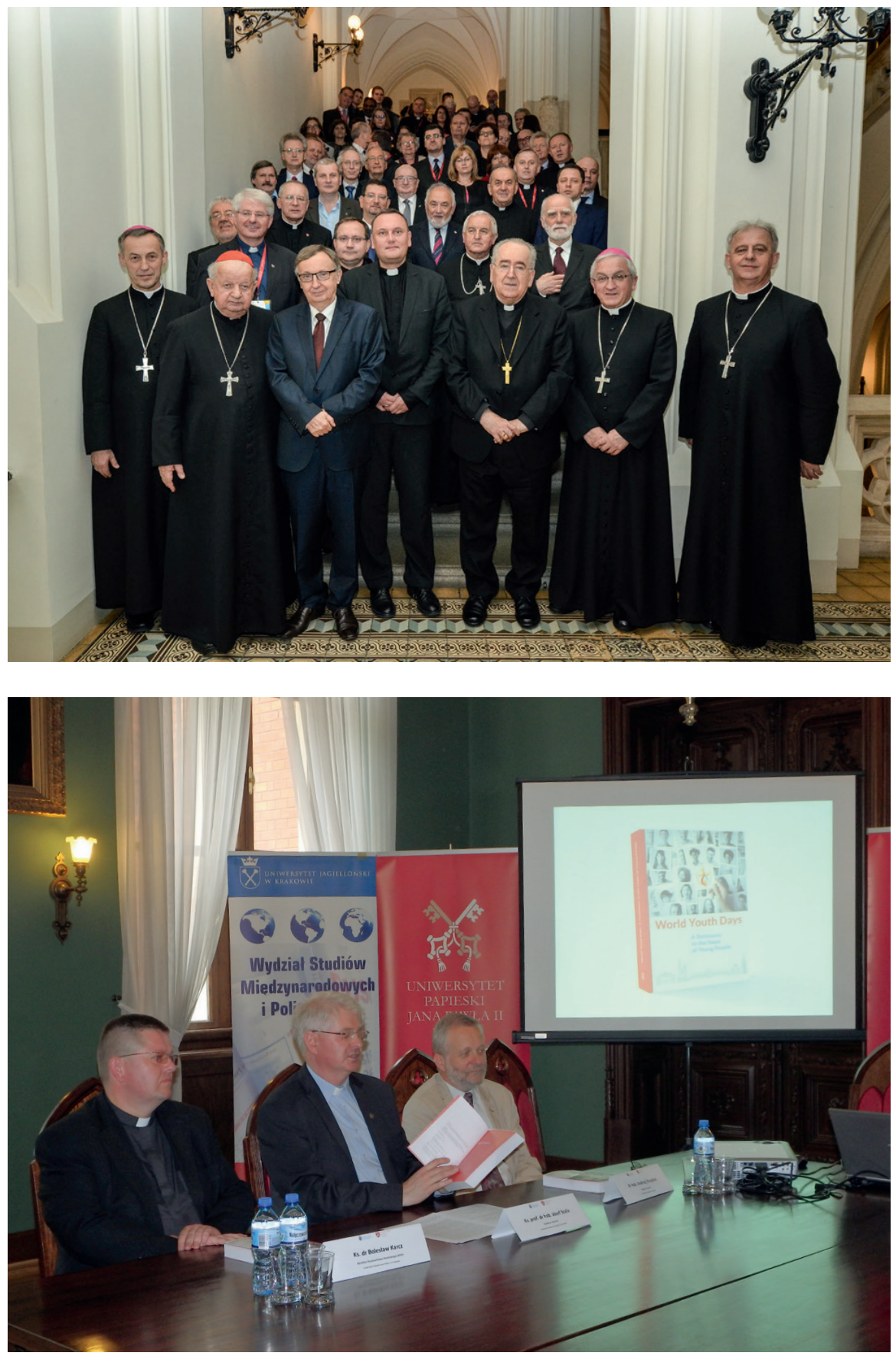